\title{
Molecular Structure and Chirality Detection by Microwave Spectroscopy
}

Simon Lobsiger, Cristobal Perez, Luca Evangelisti, Kevin K. Lehmann*, Brooks H. Pate*

Department of Chemistry, University of Virginia, McCormick Road, Charlottesville, VA 22904, USA

*Towhom correspondence should be addressed: lehmann@virginia.edu, brookspate@virginia.edu

\section{Supporting Information}

Table of contents:

1- Explicit expression for three level mixing

2- Theoretical calculation

3- Spectrum analysis

4- Measured rotational transitions and fits

5- Kraitchman analysis

6- References 


\section{Explicit Expressions for Three Level Mixing}

A. Expression for the Enantiomer-Specific Signal

We will expand the asymmetric top rotational states in a symmetric top basis where the symmetric top states are labeled $\mid J, \tau, M>$ with $\tau=0,1 \ldots 2 \mathrm{~J}$. In terms of $\mathbf{J}$ and $\tau$, the labels $K_{p}=$ Floor $(J+1+\tau / 2)$ and $K_{o}=$ Floor $(\mathrm{J}+1-\tau / 2)$.

$|J,, M\rangle={ }_{K=J}^{J} A_{K},|J, K, M\rangle$

We use the definitions of the direction cosine matrix elements, $\phi_{\alpha, G}$, given by Townes \& Schalow(1), table 4.4 , where $\alpha=a, b, c$ is one of the molecular axes and $G=X, Y, Z$ one of the laboratory axes. We can write the transition matrix elements

$$
\begin{aligned}
& \left\langle J,\left.\quad M\right|_{, G} \mid J, \quad, M\right\rangle=\langle J, \quad|\quad| J, \quad\rangle\left\langle J,\left.M\right|_{G} \mid J, M\right\rangle \\
& \langle J, \quad|\quad| J, \quad\rangle=\left\langle\left. J\right|_{J} \mid J\right\rangle_{K, K} A_{K,} A_{K},\langle J, K|\quad| J, K\rangle
\end{aligned}
$$

Consider that we first apply an X polarized microwave pulse of amplitude $\mathrm{E}_{\mathrm{ex}}$ and duration $\Delta \mathrm{t}$ on the $\mid \mathrm{J}$ ', $\tau$ ' '> -> $|\mathrm{J}, \tau\rangle$ transition for which is allowed by the a component of the dipole moment. Starting in the initial state with projection quantum number $\mathrm{M}$ ', we have following excitation:

$$
\left|\Psi_{M^{\prime \prime}}(t)\right\rangle=\cos \left(\frac{\theta\left(M^{\prime \prime}\right)}{2}\right) e^{-i E\left(J^{\prime \prime}, \tau^{\prime \prime}\right) t / \hbar}\left|J^{\prime \prime}, \tau^{\prime \prime}, M^{\prime \prime}\right\rangle+i \sin \left(\frac{\theta\left(M^{\prime \prime}\right)}{2}\right) e^{-i E(J, \tau) t / \hbar} \sum_{M=M^{\prime \prime} \pm 1} b_{M, M^{\prime \prime}}|J, \tau, M\rangle
$$


with

$$
\begin{aligned}
& \left.\theta\left(M^{\prime \prime}\right)=\frac{E_{e x} \Delta t}{\hbar} \mu_{\alpha}\left\langle J, \tau\left|\phi_{X}\right| J^{\prime \prime}, \tau^{\prime \prime}\right\rangle\right) \sqrt{\left.\left\langle J, M^{\prime \prime}+1\left|\phi_{X}\right| J^{\prime \prime}, M^{\prime \prime}\right\rangle\right|^{2}+\left|\left\langle J, M^{\prime \prime}-1\left|\phi_{X}\right| J^{\prime \prime}, M^{\prime \prime}\right\rangle\right|^{2}}
\end{aligned}
$$

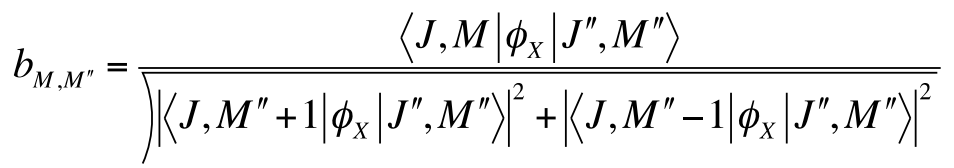

Following this excitation, we then apply resonant excitation with polarization $Z$, amplitude $\mathrm{E}_{\text {ex }}$ and pulse duration $\Delta \mathrm{t}^{\prime}$ on the $|\mathrm{J}, \tau\rangle->\left|\mathrm{J}^{\prime}, \tau^{\prime}\right\rangle$ transition which we will assume to have transition polarization $\beta$. Following this pulse, the initial state $\mid J^{\prime}, \tau^{\prime \prime}, M^{\prime \prime}>$ will be transformed into

$$
\begin{aligned}
& \left|\Psi_{M^{\prime \prime}}(t)\right\rangle=\cos \left(\frac{\theta\left(M^{\prime \prime}\right)}{2}\right) e^{-i E\left(J^{\prime \prime}, \tau^{\prime \prime}\right) t / \hbar}\left|J^{\prime \prime}, \tau^{\prime \prime}, M^{\prime \prime}\right\rangle \\
& +i \sin \left(\frac{\theta\left(M^{\prime \prime}\right)}{2}\right) e^{-i E(J, \tau) t / \hbar} \sum_{M=M^{\prime \prime} \pm 1} \cos \left(\frac{\theta^{\prime}\left(M^{\prime \prime}\right)}{2}\right) b_{M, M^{\prime \prime}}|J, \tau, M\rangle \\
& -i \sin \left(\frac{\theta\left(M^{\prime \prime}\right)}{2}\right) e^{-i E\left(J^{\prime}, \tau^{\prime}\right) t / \hbar} \sum_{M=M^{\prime \prime} \pm 1} \sin \left(\frac{\theta^{\prime}\left(M^{\prime \prime}\right)}{2}\right) b_{M, M^{\prime \prime}}\left|J^{\prime}, \tau^{\prime}, M\right\rangle
\end{aligned}
$$

with

$\theta^{\prime}(M)=\frac{E_{e x}^{\prime} \Delta t^{\prime}}{\hbar} \mu_{\beta}\left\langle J^{\prime}, \tau^{\prime}\left|\varphi_{\beta}\right| J, \tau\right\rangle\left\langle J^{\prime}, M\left|\varphi_{Z}\right| J, M\right\rangle$

This will result in a Y polarized emission (perpendicular to the other two excitation polarizations) at the resonant frequency $v_{0}=\left(\mathrm{E}\left(\mathrm{J}^{\prime}, \tau^{\prime}\right)-\mathrm{E}\left(\mathrm{J}^{\prime}, \tau^{\prime \prime}\right)\right) / \mathrm{h} . \quad \gamma$ is the polarization direction for the $\left|\mathrm{J}^{\prime}, \tau^{\prime \prime}\right\rangle->\left|\mathrm{J}^{\prime}, \tau^{\prime}\right\rangle$ transition. By the selection rules of the rotational transitions of an asymmetric rotor, we have that $\gamma$ must be perpendicular to both $\alpha$ and $\beta$. This polarization that will emit a field is given by:

$$
P_{Y}(t)=\frac{N_{J}}{2 J+1} \quad \frac{N_{J}}{2 J+1} \div{ }_{M}\left\langle\left.{ }_{M}(t)\right|_{, Y} \mid{ }_{M}(t)\right\rangle
$$




$$
\begin{aligned}
& P_{Y}=-\frac{e^{-2 \pi i v_{0} t}}{2}\left(\frac{N_{J^{\prime \prime}, \tau^{\prime \prime}}}{2 J^{\prime \prime}+1}-\frac{N_{J^{\prime}, \tau^{\prime}}}{2 J^{\prime}+1}\right) \sum_{M^{\prime \prime}, M=M^{\prime \prime} \pm 1} \sin \left(\theta\left(M^{\prime \prime}\right)\right) \sin \left(\frac{\theta^{\prime}(M)}{2}\right) b_{M, M^{\prime \prime}} \times \\
& \mu_{\gamma}\left\langle J^{\prime \prime}, \tau^{\prime \prime}\left|\varphi_{\gamma}\right| J^{\prime}, \tau^{\prime}\right\rangle\left\langle J^{\prime \prime}, M^{\prime \prime}\left|\varphi_{Y}\right| J^{\prime}, M\right\rangle+c . c .
\end{aligned}
$$

where $\mathrm{N}(\mathrm{J}, \tau)$ is the number density of molecules in the rotational state $|J, \tau\rangle$.

There are a few results that are important to the experimental implementation. One is that signal amplitude is proportional to the population difference between the states initially driven, not the final ones, where the detection is made. Thus, it is advantageous to make this the highest frequency transition. Second, regardless of drive amplitude, the signal is proportional the transition dipole matrix element for the transition at the detection frequency. Assuming one has sufficient microwave power to drive the $\pi / 2$ and $\pi$ pulses before sufficient dephasing occurs, it is best to take the detection transition to have the strongest dipole matrix element. It is also noted that changing the sign of $\mu_{\alpha}$ changes the $\operatorname{sign}$ of $\sin \left(\theta\left(M^{\prime \prime}\right)\right)$; likewise changing the sign of $\mu_{\beta}$ changes the sign of $\sin \left(\theta^{\prime}(\mathrm{M}) / 2\right)$. Thus, the sign of the overall signal is dependent upon the sign of the product $\mu_{\alpha} \mu_{\beta} \mu_{\gamma}$.(2) The signs of the individual dipole components is dependent upon how we align the inertial axes with the molecule. We are free, for example, to choose $\mu_{\alpha}$ and $\mu_{\beta}$ to be positive. However, once we have made that choice, the direction of $\mu_{\gamma}$ by the requirement that our inertial axis system is a right handed coordinate system. For a chiral molecule, the two enantiomers have opposite sign for the product $\mu_{\alpha} \mu_{\beta} \mu_{\gamma}$.(3) This can be demonstrated by considering the parity operation (in the molecular frame), which converts one enantiomer to the other and changes the sign of each component of the electric dipole moment. Lastly, we note that the chiral polarization is real, i.e. in or out of phase with the $\omega-\omega^{\prime}$ component of the product $\mathrm{E}(\mathrm{t}) \mathrm{E}^{\prime}(\mathrm{t})$. The emitted field that is detected has an additional phase shift of $90^{\circ}(4)$ and thus the emitted electric field generated by the chiral molecules will be either plus or minus $90^{\circ}$ with respect to $\omega-\omega^{\prime}$ component of the product $E(t) E^{\prime}(t)$.

\section{B. Numerical Modeling for the Solketal Measurements}

Explicitly calculating the "flip angles" that optimize the chiral signal, for the $\left|1_{01}\right\rangle->\left|2_{11}\right\rangle->\left|2_{02}\right\rangle$ excitation sequence shown in Fig. 2 of the paper, it is found that chiral signal is optimized when $\left(\mu_{\mathrm{c}} \mathrm{E}_{0}\right.$ $\Delta \mathrm{t}) / \hbar=1.221 \pi$ and $\left(\mu_{\mathrm{b}} \mathrm{E}_{0}^{\prime} \Delta \mathrm{t}^{\prime}\right) / \hbar=1.924 \pi$. Note that these values are the prefactors in determining the Rabi flip angles, eqs. (1.4) and (1.6) and the effective "flip angle" includes the contribution of the direction cosine matrix elements.

The conditions for the optimal chiral signal can be compared to the conditions for achieving the optimum signal when directly measuring a rotational transition. These are the comparisons shown in Fig. 3 of the paper. The polarization is optimized for the $\left|1_{01}\right\rangle->\mid 2_{11}>$ when $\left(\mu_{c} E_{0} \Delta t\right) / \hbar=1.212 \pi$. Therefore, the signals observed in the direct observation of this transition can be used to optimally set the pulse duration 
for the chiral signal since there is little difference between the optimal signal conditions. For the $\left|2_{11}\right\rangle$-> $\mid 2_{02}>$, the polarization is maximum for $\left(\mu_{\mathrm{b}} \mathrm{E}_{0}^{\prime} \Delta \mathrm{t}^{\prime} / \hbar\right)=0.956 \pi$ and zero for $\left(\mu_{\mathrm{b}} \mathrm{E}^{\prime}{ }_{0} \Delta \mathrm{t}^{\prime} / \hbar\right)=2.011 \pi$. In this case, pulse duration for an effective $\pi$-pulse, defined as the pulse duration where the signal is zero is approximately twice as long as the duration for the optimal signal. The basic concept of the $\pi / 2$ - and $\pi$ pulses is valid for these measurements. Furthermore, using the pulse duration that gives a signal minimum in the single-photon measurement, the effective $\pi$-pulse where $\left(\mu_{\mathrm{b}} \mathrm{E}_{0}^{\prime} \Delta \mathrm{t}^{\prime} / \hbar\right)=2.011 \pi$, provides an excellent estimate of the pulse duration that should be used in the chiral three wave mixing experiment to achieve optimum signal $\left(\left(\mu_{\mathrm{b}} \mathrm{E}^{\prime}{ }_{0} \Delta \mathrm{t}^{\prime}\right) / \hbar=1.924 \pi\right)$.

The optimized magnitude of the chiral signal is $82 \%$ of the emission when the $\left.\left|1_{01}>->\right| 2_{02}\right\rangle$ transition is driven by a pulse of optimal length. This calculation ignores the Boltzmann population factors, which favor the chiral signal. The observed signals in Fig. 4 of the paper are quite a bit weaker than this estimate, and that is most certainly the result of phase mismatch. The field on the detector emitted by the sample at different points in space have a relative phase of

$$
\left(\omega X+\omega^{\prime} Y-\left(\omega-\omega^{\prime}\right) X\right) / c=c \omega^{\prime}(X+Y)
$$

where the position of the emitter is given by (X,Y,Z). For $\omega^{\prime}=2 \pi \cdot 1839 \mathrm{MHz}$, emission will be out of phase for molecules with $(\Delta \mathrm{X}+\Delta \mathrm{Y})=8 \mathrm{~cm}$ (assuming that the excitation waves have planer wavefronts). However, if we consider the motion of the emitters between excitation and detection, we have the molecules initially as position $(\mathrm{X}, \mathrm{Y})$ will have a phase

$$
\left[\omega^{\prime}(X+Y)-\left(\omega-\omega^{\prime}\right) V_{X} t\right] / c
$$

If we consider that the mean value of $\mathrm{V}_{\mathrm{X}}$ will be positively correlated with the value of $\mathrm{X}$ we can anticipate that the spatial dephasing will initially decrease as time increases. This rationalizes the observation that the signal chiral initially grows with time and then decreases more slowly than the normal FID signal. 


\section{Theoretical calculations}

The structure of solketal was optimized with the M06-2X density functional and the 6-311++G(d,p) basis set using Gaussian09 (5). The Cartesian coordinates in the principal axes system are given in Table S1. Calculated rotational constants and dipole moment components are given in Table S2.

Table S1. Cartesian coordinates (in $\AA$ ) in the principal axes system of the M06-2X/6-311++G(d,p) optimized structure of solketal.

\begin{tabular}{lrrr}
\hline Atom & $\mathrm{a}$ & \multicolumn{1}{c}{$\mathrm{b}$} & $\mathrm{c}$ \\
\hline $\mathrm{C}$ & -0.887634 & 0.666086 & -0.491933 \\
$\mathrm{C}$ & 1.177658 & -0.294758 & 0.016540 \\
$\mathrm{H}$ & -1.256553 & 1.018295 & -1.458003 \\
$\mathrm{O}$ & -0.039632 & -0.459370 & -0.721373 \\
$\mathrm{O}$ & 0.959103 & 0.817090 & 0.865978 \\
$\mathrm{C}$ & 1.419311 & -1.516044 & 0.876911 \\
$\mathrm{H}$ & 1.534787 & -2.396534 & 0.243289 \\
$\mathrm{H}$ & 2.325432 & -1.381447 & 1.469406 \\
$\mathrm{H}$ & 0.571342 & -1.661363 & 1.547117 \\
$\mathrm{C}$ & 2.310918 & -0.023811 & -0.961784 \\
$\mathrm{H}$ & 3.248924 & 0.106874 & -0.420021 \\
$\mathrm{H}$ & 2.407735 & -0.860846 & -1.654739 \\
$\mathrm{H}$ & 2.105862 & 0.877940 & -1.541249 \\
$\mathrm{C}$ & -2.046921 & 0.223262 & 0.387828 \\
$\mathrm{H}$ & -1.659390 & -0.012215 & 1.389151 \\
$\mathrm{H}$ & -2.779118 & 1.029103 & 0.480156 \\
$\mathrm{O}$ & -2.712929 & -0.882725 & -0.175339 \\
$\mathrm{H}$ & -2.031865 & -1.520331 & -0.416673 \\
$\mathrm{C}$ & 0.036169 & 1.662768 & 0.205937 \\
$\mathrm{H}$ & -0.470987 & 2.267339 & 0.958963 \\
$\mathrm{H}$ & 0.540534 & 2.322201 & -0.508495 \\
\hline
\end{tabular}

Table S2. M06-2X/6-311++g(d,p) calculated rotational constants and dipole moment components of (R)and (S)-solketal.

\begin{tabular}{lrr}
\hline & \multicolumn{2}{c}{ full ${ }^{12} \mathrm{C} /{ }^{16} \mathrm{O} /{ }^{1} \mathrm{H}$} \\
\hline $\mathrm{A} / \mathrm{MHz}$ & \multicolumn{2}{c}{2756.30} \\
$\mathrm{~B} / \mathrm{MHz}$ & \multicolumn{2}{c}{1247.66} \\
$\mathrm{C} / \mathrm{MHz}$ & \multicolumn{2}{c}{1088.30} \\
& \multicolumn{3}{c}{$(\mathrm{S})$} & $(\mathrm{R})$ \\
& 1.8 & -1.8 \\
$\mu_{\mathrm{a}} / \mathrm{D}$ & 1.2 & 1.2 \\
$\mu_{\mathrm{b}} / \mathrm{D}$ & -0.2 & -0.2 \\
$\mu_{\mathrm{c}} / \mathrm{D}$ & -0.2 \\
\hline
\end{tabular}




\section{Spectrum analysis}

Initial rigid rotor fits of the broadband spectra were performed using the JB95 program package which is freely available from the NIST website (6). Refined fits were obtained using the AABS (7) package, which allows graphical analysis of rotational resolved broadband spectra and integrates the prediction and fitting algorithms of Pickett's SPCAT/SPFIT packages $(8,9)$. The Kraitchman substitution structure $\left(\mathrm{r}_{\mathrm{s}}\right)$ for solketal was calculated using the KRA program package (10). All programs, expect JB95, are freely available from the PROSPE website (10). The UCSF Chimera package (11) was used for visualization of molecular structures. 


\section{Measured Rotational Transitions and Fits}

Table S3. Measured rotational transitions ( $v_{\text {obs }}$ ) of the solketal ${ }^{12} \mathrm{C} /{ }^{16} \mathrm{O} /{ }^{1} \mathrm{H}$ isotopologue and residuals $\left(v_{\text {obs }}-v_{\text {calc }}\right)$ (frequencies in $\mathrm{MHz}$ ).

\begin{tabular}{|c|c|c|c|c|c|c|c|c|}
\hline $\mathrm{J}^{\prime}$ & $\mathrm{K}_{\mathrm{a}}^{\prime}$ & $\mathrm{K}_{\mathrm{c}}{ }^{\prime}$ & $\leftarrow$ & $\mathrm{J}^{\prime \prime}$ & $\mathrm{K}_{\mathrm{a}}{ }^{\prime \prime}$ & $\mathrm{K}_{\mathrm{c}}{ }^{\prime \prime}$ & $v_{\text {obs }}$ & $V_{\text {obs }}-V_{\text {calc }}$ \\
\hline 3 & 1 & 2 & & 3 & 0 & 3 & 2107.2863 & 0.0036 \\
\hline 1 & 0 & 1 & & 0 & 0 & 0 & 2307.5191 & 0.0010 \\
\hline 4 & 1 & 3 & & 4 & 0 & 4 & 2499.7472 & -0.0150 \\
\hline 5 & 2 & 4 & & 4 & 3 & 1 & 3489.0401 & -0.0023 \\
\hline 4 & 1 & 4 & & 3 & 2 & 1 & 3586.0727 & 0.0089 \\
\hline 1 & 1 & 1 & & 0 & 0 & 0 & 3827.2208 & -0.0064 \\
\hline 1 & 1 & 0 & & 0 & 0 & 0 & 3981.3652 & -0.0009 \\
\hline 6 & 2 & 4 & & 6 & 1 & 5 & 3985.8288 & 0.0154 \\
\hline 4 & 2 & 2 & & 4 & 1 & 3 & 4205.4280 & 0.0174 \\
\hline 8 & 2 & 6 & & 8 & 1 & 7 & 4218.4244 & -0.0074 \\
\hline 3 & 2 & 1 & & 3 & 1 & 2 & 4390.4550 & -0.0019 \\
\hline 2 & 1 & 2 & & 1 & 1 & 1 & 4460.8878 & 0.0003 \\
\hline 7 & 1 & 6 & & 7 & 0 & 7 & 4590.2209 & 0.0047 \\
\hline 2 & 0 & 2 & & 1 & 0 & 1 & 4603.8861 & -0.0003 \\
\hline 2 & 1 & 1 & & 1 & 1 & 0 & 4769.1702 & 0.0045 \\
\hline 2 & 2 & 1 & & 2 & 1 & 2 & 5021.5270 & -0.0021 \\
\hline 3 & 2 & 2 & & 3 & 1 & 3 & 5259.4731 & 0.0087 \\
\hline 3 & 0 & 3 & & 2 & 1 & 2 & 5501.6318 & 0.0047 \\
\hline 4 & 2 & 3 & & 4 & 1 & 4 & 5579.9163 & 0.0092 \\
\hline 6 & 2 & 5 & & 5 & 3 & 2 & 5702.6803 & -0.0107 \\
\hline 2 & 1 & 2 & & 1 & 0 & 1 & 5980.5885 & -0.0081 \\
\hline 5 & 2 & 4 & & 5 & 1 & 5 & 5984.2288 & -0.0078 \\
\hline 8 & 3 & 6 & & 8 & 2 & 6 & 6683.9681 & -0.0035 \\
\hline 3 & 1 & 3 & & 2 & 1 & 2 & 6684.5816 & 0.0030 \\
\hline 3 & 0 & 3 & & 2 & 0 & 2 & 6878.3329 & -0.0042 \\
\hline 3 & 2 & 2 & & 2 & 2 & 1 & 6922.5256 & 0.0119 \\
\hline 3 & 2 & 1 & & 2 & 2 & 0 & 6966.7038 & 0.0074 \\
\hline 3 & 1 & 2 & & 2 & 1 & 1 & 7146.4879 & -0.0046 \\
\hline 7 & 2 & 6 & & 6 & 3 & 3 & 7848.4095 & 0.0082 \\
\hline 3 & 3 & 0 & & 3 & 2 & 1 & 7935.7335 & 0.0030 \\
\hline 4 & 0 & 4 & & 3 & 1 & 3 & 7938.9567 & -0.0061 \\
\hline 3 & 3 & 1 & & 3 & 2 & 2 & 7990.5448 & -0.0044 \\
\hline 3 & 1 & 2 & & 2 & 2 & 1 & 2587.3887 & 0.0081 \\
\hline 2 & 0 & 2 & & 1 & 1 & 1 & 3084.1856 & 0.0083 \\
\hline 3 & 2 & 2 & & 3 & 1 & 2 & 4335.1409 & 0.0077 \\
\hline 2 & 2 & 1 & & 2 & 1 & 1 & 4559.1044 & -0.0075 \\
\hline 2 & 2 & 0 & & 2 & 1 & 1 & 4570.2390 & -0.0140 \\
\hline 3 & 0 & 3 & & 2 & 1 & 1 & 5039.2016 & -0.0082 \\
\hline 4 & 2 & 2 & & 4 & 1 & 4 & 5743.2789 & -0.0048 \\
\hline 3 & 2 & 2 & & 3 & 0 & 3 & 6442.4230 & 0.0072 \\
\hline 2 & 1 & 1 & & 1 & 0 & 1 & 6443.0180 & 0.0042 \\
\hline 6 & 2 & 5 & & 6 & 0 & 6 & 7012.9018 & -0.0027 \\
\hline \multirow[t]{2}{*}{4} & 0 & 4 & & 3 & 1 & 2 & 7014.6171 & -0.0145 \\
\hline & & & & & & & RMS & $7.88 \mathrm{kHz}$ \\
\hline
\end{tabular}


Table S4. Measured rotational transitions ( $v_{\text {obs }}$ ) of the solketal single ${ }^{13} \mathrm{C}(1)$ isotopologue and residuals $\left(v_{\text {obs }}-v_{\text {calc }}\right)$ (frequencies in MHz).

\begin{tabular}{|c|c|c|c|c|c|c|c|c|}
\hline $\mathrm{J}^{\prime}$ & $\mathrm{K}_{\mathrm{a}}^{\prime}$ & $\mathrm{K}_{\mathrm{c}}^{\prime}$ & $\leftarrow$ & $\mathrm{J}^{\prime \prime}$ & $\mathrm{K}_{\mathrm{a}}^{\prime \prime}$ & $\mathrm{K}_{\mathrm{c}}^{\prime \prime}$ & $v_{\mathrm{obs}}$ & $v_{\text {obs }}-v_{\text {calc }}$ \\
\hline 1 & 1 & 1 & & 0 & 0 & 0 & 3779.8907 & -0.0028 \\
\hline 3 & 2 & 1 & & 3 & 1 & 2 & 4264.5851 & 0.0003 \\
\hline 2 & 1 & 2 & & 1 & 1 & 1 & 4442.2356 & 0.0002 \\
\hline 2 & 0 & 2 & & 1 & 0 & 1 & 4590.2209 & -0.0010 \\
\hline 2 & 1 & 1 & & 1 & 1 & 0 & 4762.8965 & 0.0000 \\
\hline 3 & 0 & 3 & & 2 & 1 & 2 & 5524.3022 & -0.0062 \\
\hline 2 & 1 & 2 & & 1 & 0 & 1 & 5920.8372 & -0.0039 \\
\hline 3 & 1 & 3 & & 2 & 1 & 2 & 6655.8898 & 0.0007 \\
\hline 3 & 0 & 3 & & 2 & 0 & 2 & 6854.9227 & -0.0050 \\
\hline 3 & 2 & 2 & & 2 & 2 & 1 & 6903.8381 & 0.0152 \\
\hline 3 & 2 & 1 & & 2 & 2 & 0 & 6952.7277 & 0.0037 \\
\hline \multirow[t]{2}{*}{3} & 1 & 2 & & 2 & 1 & 1 & 7136.2816 & -0.0043 \\
\hline & & & & & & & RMS & $5.45 \mathrm{kHz}$ \\
\hline
\end{tabular}

Table S5. Measured rotational transitions ( $\left.v_{\text {obs }}\right)$ of the solketal single ${ }^{13} \mathrm{C}(2)$ isotopologue and residuals $\left(v_{\text {obs }}-v_{\text {calc }}\right)$ (frequencies in $\mathrm{MHz}$ ).

\begin{tabular}{|c|c|c|c|c|c|c|c|c|}
\hline $\mathrm{J}^{\prime}$ & $\mathrm{K}_{\mathrm{a}}{ }^{\prime}$ & $\mathrm{K}_{\mathrm{c}}^{\prime}$ & $\leftarrow$ & $\mathrm{J}^{\prime \prime}$ & $\mathrm{K}_{\mathrm{a}}{ }^{\prime \prime}$ & $\mathrm{K}_{\mathrm{c}}{ }^{\prime \prime}$ & $v_{\text {obs }}$ & $V_{\text {obs }}-V_{\text {calc }}$ \\
\hline 2 & 1 & 2 & & 1 & 1 & 1 & 4449.8447 & -0.0022 \\
\hline 2 & 0 & 2 & & 1 & 0 & 1 & 4592.6026 & -0.0017 \\
\hline 2 & 1 & 1 & & 1 & 1 & 0 & 4757.6786 & 0.0019 \\
\hline 3 & 0 & 3 & & 2 & 1 & 2 & 5491.4381 & -0.0020 \\
\hline 2 & 1 & 2 & & 1 & 0 & 1 & 5962.5364 & -0.0014 \\
\hline 3 & 1 & 3 & & 2 & 1 & 2 & 6668.0093 & 0.0013 \\
\hline 3 & 0 & 3 & & 2 & 0 & 2 & 6861.3652 & -0.0084 \\
\hline 3 & 2 & 2 & & 2 & 2 & 1 & 6905.6279 & 0.0113 \\
\hline 3 & 2 & 1 & & 2 & 2 & 0 & 6949.8750 & 0.0093 \\
\hline \multirow[t]{2}{*}{3} & 1 & 2 & & 2 & 1 & 1 & 7129.2376 & -0.0092 \\
\hline & & & & & & & RMS & $6.27 \mathrm{kHz}$ \\
\hline
\end{tabular}

Table S6. Measured rotational transitions ( $\left.v_{\text {obs }}\right)$ of the solketal single ${ }^{13} \mathrm{C}(3)$ isotopologue and residuals $\left(v_{\text {obs }}-v_{\text {calc }}\right)$ (frequencies in $\mathrm{MHz}$ ).

\begin{tabular}{|c|c|c|c|c|c|c|c|c|}
\hline $\mathrm{J}^{\prime}$ & $\mathrm{K}_{\mathrm{a}}^{\prime}$ & $\mathrm{K}_{\mathrm{c}}^{\prime}$ & $\leftarrow$ & $\mathrm{J}^{\prime \prime}$ & $\mathrm{K}_{\mathrm{a}}{ }^{\prime \prime}$ & $\mathrm{K}_{\mathrm{c}}{ }^{\prime \prime}$ & $v_{\text {obs }}$ & $V_{\text {obs }}-V_{\text {calc }}$ \\
\hline 2 & 1 & 2 & & 1 & 1 & 1 & 4446.5386 & -0.0007 \\
\hline 2 & 0 & 2 & & 1 & 0 & 1 & 4588.8733 & -0.0033 \\
\hline 2 & 1 & 1 & & 1 & 1 & 0 & 4753.2291 & 0.0015 \\
\hline 2 & 1 & 2 & & 1 & 0 & 1 & 5969.3804 & 0.0004 \\
\hline 3 & 1 & 3 & & 2 & 1 & 2 & 6663.1366 & 0.0011 \\
\hline 3 & 0 & 3 & & 2 & 0 & 2 & 6856.1364 & -0.0093 \\
\hline 3 & 2 & 2 & & 2 & 2 & 1 & 6899.8090 & 0.0098 \\
\hline 3 & 2 & 1 & & 2 & 2 & 0 & 6943.4649 & 0.0062 \\
\hline \multirow[t]{2}{*}{3} & 1 & 2 & & 2 & 1 & 1 & 7122.6675 & -0.0064 \\
\hline & & & & & & & RMS & $5.59 \mathrm{kHz}$ \\
\hline
\end{tabular}


Table S7. Measured rotational transitions ( $\left.v_{\text {obs }}\right)$ of the solketal single ${ }^{13} \mathrm{C}(4)$ isotopologue and residuals $\left(v_{\text {obs }}-v_{\text {calc }}\right)$ (frequencies in MHz).

\begin{tabular}{|c|c|c|c|c|c|c|c|c|}
\hline $\mathrm{J}^{\prime}$ & $\mathrm{K}_{\mathrm{a}}^{\prime}$ & $\mathrm{K}_{\mathrm{c}}^{\prime}$ & $\leftarrow$ & $\mathrm{J}^{\prime \prime}$ & $\mathrm{K}_{\mathrm{a}}^{\prime \prime}$ & $\mathrm{K}_{\mathrm{c}}^{\prime \prime}$ & $v_{\mathrm{obs}}$ & $V_{\text {obs }}-V_{\text {calc }}$ \\
\hline 2 & 1 & 2 & & 1 & 1 & 1 & 4422.6384 & 0.0054 \\
\hline 2 & 0 & 2 & & 1 & 0 & 1 & 4566.6760 & 0.0020 \\
\hline 2 & 1 & 1 & & 1 & 1 & 0 & 4733.9517 & 0.0076 \\
\hline 3 & 0 & 3 & & 2 & 1 & 2 & 5481.5721 & -0.0088 \\
\hline 2 & 1 & 2 & & 1 & 0 & 1 & 5906.4611 & -0.0064 \\
\hline 3 & 1 & 3 & & 2 & 1 & 2 & 6626.9251 & 0.0074 \\
\hline 3 & 0 & 3 & & 2 & 0 & 2 & 6821.3726 & -0.0018 \\
\hline 3 & 2 & 2 & & 2 & 2 & 1 & 6867.4207 & 0.0140 \\
\hline 3 & 2 & 1 & & 2 & 2 & 0 & 6913.4468 & 0.0018 \\
\hline 3 & 1 & 2 & & 2 & 1 & 1 & 7093.3359 & -0.0062 \\
\hline 4 & 0 & 4 & & 3 & 1 & 3 & 7898.7124 & -0.0045 \\
\hline 3 & 1 & 3 & & 2 & 0 & 2 & 7966.7050 & -0.0061 \\
\hline & & & & & & & RMS & $6.89 \mathrm{kHz}$ \\
\hline
\end{tabular}

Table S8. Measured rotational transitions ( $\left.v_{\text {obs }}\right)$ of the solketal single ${ }^{13} \mathrm{C}(5)$ isotopologue and residuals $\left(v_{\text {obs }}-v_{\text {calc }}\right)$ (frequencies in $\mathrm{MHz}$ ).

\begin{tabular}{|c|c|c|c|c|c|c|c|c|}
\hline $\mathrm{J}^{\prime}$ & $\mathrm{K}_{\mathrm{a}}^{\prime}$ & $\mathrm{K}_{\mathrm{c}}^{\prime}$ & $\leftarrow$ & $\mathrm{J}^{\prime \prime}$ & $\mathrm{K}_{\mathrm{a}}{ }^{\prime \prime}$ & $\mathrm{K}_{\mathrm{c}}{ }^{\prime}$ & $\nu_{\text {obs }}$ & $v_{\text {obs }}-v_{\text {calc }}$ \\
\hline 2 & 1 & 2 & & 1 & 1 & 1 & 4406.1420 & -0.0006 \\
\hline 2 & 0 & 2 & & 1 & 0 & 1 & 4543.6748 & -0.0009 \\
\hline 2 & 1 & 1 & & 1 & 1 & 0 & 4701.6751 & 0.0063 \\
\hline 2 & 1 & 2 & & 1 & 0 & 1 & 5930.4357 & -0.0150 \\
\hline 3 & 1 & 3 & & 2 & 1 & 2 & 6603.0079 & 0.0052 \\
\hline 3 & 0 & 3 & & 2 & 0 & 2 & 6790.2353 & -0.0015 \\
\hline 3 & 2 & 2 & & 2 & 2 & 1 & 6830.8477 & 0.0152 \\
\hline 3 & 2 & 1 & & 2 & 2 & 0 & 6871.4411 & 0.0070 \\
\hline 3 & 1 & 2 & & 2 & 1 & 1 & 7045.8466 & -0.0026 \\
\hline 4 & 0 & 4 & & 3 & 1 & 3 & 7808.7056 & -0.0141 \\
\hline \multirow[t]{2}{*}{3} & 1 & 3 & & 2 & 0 & 2 & 7989.7801 & 0.0024 \\
\hline & & & & & & & RMS & $8.50 \mathrm{kHz}$ \\
\hline
\end{tabular}

Table S9. Measured rotational transitions ( $\left.v_{\text {obs }}\right)$ of the solketal single ${ }^{13} \mathrm{C}(6)$ isotopologue and residuals $\left(v_{\text {obs }}-v_{\text {calc }}\right)$ (frequencies in $\mathrm{MHz}$ ).

\begin{tabular}{|c|c|c|c|c|c|c|c|c|}
\hline $\mathrm{J}^{\prime}$ & $\mathrm{K}_{\mathrm{a}}^{\prime}$ & $\mathrm{K}_{\mathrm{c}}^{\prime}$ & $\leftarrow$ & $\mathrm{J}^{\prime \prime}$ & $\mathrm{K}_{\mathrm{a}}{ }^{\prime \prime}$ & $\mathrm{K}_{\mathrm{c}}{ }^{\prime \prime}$ & $v_{\text {obs }}$ & $V_{\text {obs }}-v_{\text {calc }}$ \\
\hline 2 & 1 & 2 & & 1 & 1 & 1 & 4419.0677 & -0.0018 \\
\hline 2 & 0 & 2 & & 1 & 0 & 1 & 4559.3338 & -0.0027 \\
\hline 2 & 1 & 1 & & 1 & 1 & 0 & 4720.8456 & 0.0018 \\
\hline 3 & 0 & 3 & & 2 & 1 & 2 & 5423.3115 & 0.0016 \\
\hline 2 & 1 & 2 & & 1 & 0 & 1 & 5948.8006 & -0.0021 \\
\hline 3 & 1 & 3 & & 2 & 1 & 2 & 6622.1614 & 0.0010 \\
\hline 3 & 0 & 3 & & 2 & 0 & 2 & 6812.7695 & -0.0065 \\
\hline 3 & 2 & 2 & & 2 & 2 & 1 & 6854.9227 & 0.0138 \\
\hline 3 & 2 & 1 & & 2 & 2 & 0 & 6897.0535 & 0.0057 \\
\hline 3 & 1 & 2 & & 2 & 1 & 1 & 7074.3482 & -0.0063 \\
\hline \multirow[t]{2}{*}{4} & 0 & 4 & & 3 & 1 & 3 & 7837.8074 & -0.0045 \\
\hline & & & & & & & RMS & $5.63 \mathrm{kHz}$ \\
\hline
\end{tabular}


Table S10. Measured rotational transitions ( $\left.v_{\mathrm{obs}}\right)$ of the solketal single ${ }^{18} \mathrm{O}(1)$ isotopologue and residuals $\left(v_{\text {obs }}-v_{\text {calc }}\right)$ (frequencies in $\mathrm{MHz}$ ).

\begin{tabular}{|c|c|c|c|c|c|c|c|c|}
\hline $\mathrm{J}^{\prime}$ & $\mathrm{K}_{\mathrm{a}}^{\prime}$ & $\mathrm{K}_{\mathrm{c}}^{\prime}$ & $\leftarrow$ & $\mathrm{J}^{\prime \prime}$ & $\mathrm{K}_{\mathrm{a}}^{\prime \prime}$ & $\mathrm{K}_{\mathrm{c}}^{\prime \prime}$ & $v_{\mathrm{obs}}$ & $V_{\text {obs }}-V_{\text {calc }}$ \\
\hline 2 & 1 & 2 & & 1 & 1 & 1 & 4428.7661 & -0.0124 \\
\hline 2 & 0 & 2 & & 1 & 0 & 1 & 4569.3713 & 0.0000 \\
\hline 2 & 1 & 1 & & 1 & 1 & 0 & 4731.9653 & 0.0005 \\
\hline 3 & 1 & 3 & & 2 & 1 & 2 & 6636.5053 & 0.0048 \\
\hline 3 & 0 & 3 & & 2 & 0 & 2 & 6826.9154 & 0.0034 \\
\hline 3 & 2 & 1 & & 2 & 2 & 0 & 6914.1602 & 0.0034 \\
\hline 3 & 1 & 2 & & 2 & 1 & 1 & 7090.7769 & -0.0037 \\
\hline
\end{tabular}

Table S11. Measured rotational transitions $\left(v_{\text {obs }}\right)$ of the solketal single ${ }^{18} \mathrm{O}(2)$ isotopologue and residuals $\left(v_{\text {obs }}-v_{\text {calc }}\right)$ (frequencies in $\mathrm{MHz}$ ).

\begin{tabular}{|c|c|c|c|c|c|c|c|c|}
\hline $\mathrm{J}^{\prime}$ & $\mathrm{K}_{\mathrm{a}}^{\prime}$ & $\mathrm{K}_{\mathrm{c}}^{\prime}$ & $\leftarrow$ & $\mathrm{J}^{\prime \prime}$ & $\mathrm{K}_{\mathrm{a}}{ }^{\prime \prime}$ & $\mathrm{K}_{\mathrm{c}}{ }^{\prime \prime}$ & $v_{\text {obs }}$ & $V_{\text {obs }}-V_{\text {calc }}$ \\
\hline 3 & 1 & 3 & & 2 & 1 & 2 & 6676.4809 & -0.0028 \\
\hline 3 & 0 & 3 & & 2 & 0 & 2 & 6867.7976 & 0.0072 \\
\hline 3 & 2 & 1 & & 2 & 2 & 0 & 6955.0372 & 0.0059 \\
\hline 3 & 1 & 2 & & 2 & 1 & 1 & 7132.5476 & -0.0046 \\
\hline 2 & 1 & 2 & & 1 & 1 & 1 & 4455.4285 & -0.0056 \\
\hline \multirow[t]{2}{*}{2} & 1 & 1 & & 1 & 1 & 0 & 4759.8082 & -0.0029 \\
\hline & & & & & & & RMS & $5.11 \mathrm{kHz}$ \\
\hline
\end{tabular}

Table S12. Measured rotational transitions ( $\left.v_{\mathrm{obs}}\right)$ of the solketal single ${ }^{18} \mathrm{O}(3)$ isotopologue and residuals $\left(v_{\text {obs }}-v_{\text {calc }}\right)$ (frequencies in MHz).

\begin{tabular}{ccccccccr}
\hline $\mathrm{J}^{\prime}$ & $\mathrm{K}_{\mathrm{a}}{ }^{\prime}$ & $\mathrm{K}_{\mathrm{c}}{ }^{\prime}$ & $\leftarrow$ & $\mathrm{J}^{\prime \prime}$ & $\mathrm{K}_{\mathrm{a}}{ }^{\prime \prime}$ & $\mathrm{K}_{\mathrm{c}}{ }^{\prime \prime}$ & $v_{\text {obs }}$ & $v_{\text {obs }}-v_{\text {calc }}$ \\
\hline 2 & 1 & 2 & & 1 & 1 & 1 & 4309.0245 & 0.0067 \\
2 & 0 & 2 & & 1 & 0 & 1 & 4446.0915 & 0.0055 \\
2 & 1 & 1 & & 1 & 1 & 0 & 4603.2164 & -0.0122 \\
3 & 1 & 3 & & 2 & 1 & 2 & 6457.4166 & -0.0126 \\
3 & 0 & 3 & & 2 & 0 & 2 & 6644.3193 & 0.0004 \\
3 & 2 & 2 & & 2 & 2 & 1 & 6684.1709 & 0.0121 \\
\hline \multicolumn{7}{c}{} \\
\hline
\end{tabular}


Table S13. Experimental rotational parameters of the solketal ${ }^{12} \mathrm{C} /{ }^{16} \mathrm{O} /{ }^{1} \mathrm{H}$ isotopologue.

\begin{tabular}{lc}
\hline & ${ }^{12} \mathrm{C} /{ }^{16} \mathrm{O} /{ }^{1} \mathrm{H}$ \\
\hline $\mathrm{A} / \mathrm{MHz}$ & $2750.5399(15)$ \\
$\mathrm{B} / \mathrm{MHz}$ & $1230.82538(66)$ \\
$\mathrm{C} / \mathrm{MHz}$ & $1076.69416(58)$ \\
& \\
$\Delta_{\mathrm{J}} / \mathrm{kHz}$ & $0.359(12)$ \\
$\Delta_{\mathrm{JK}} / \mathrm{kHz}$ & $0.254(29)$ \\
$\Delta_{\mathrm{K}} / \mathrm{kHz}$ & $1.02(15)$ \\
$\delta_{\mathrm{J}} / \mathrm{kHz}$ & $-0.0132(39)$ \\
$\delta_{\mathrm{K}} / \mathrm{kHz}$ & $-1.88(10)$ \\
& \\
$\mathrm{N}_{\text {lines }} \mathrm{c}$ & 43 \\
$\sigma_{\text {fit }} / \mathrm{kHz}^{\mathrm{d}}$ & 7.88 \\
\hline $\mathrm{A}, \mathrm{B}, \mathrm{Cnd}$
\end{tabular}

${ }^{\mathrm{a}} \mathrm{A}, \mathrm{B}$, and $\mathrm{C}$ are rotational constants and $\Delta_{\mathrm{J}}, \Delta_{\mathrm{JK}}, \Delta_{\mathrm{K}}, \delta_{\mathrm{J}}, \delta_{\mathrm{K}}$ are quartic centrifugal distortion constants in Watson's A-reduced asymmetric rotor Hamiltonian.

${ }^{\mathrm{b}}$ Errors in parentheses are standard errors in units of the last digit.

$c$ The number of fitted lines.

${ }^{\mathrm{d}}$ Standard deviation of the fit.

Table S14. Experimental rotational parameters of the solketal single ${ }^{13} \mathrm{C}$ isotopologues.

\begin{tabular}{lcccccc}
\hline & ${ }^{13} \mathrm{C}(1)$ & ${ }^{13} \mathrm{C}(2)$ & ${ }^{13} \mathrm{C}(3)$ & ${ }^{13} \mathrm{C}(4)$ & ${ }^{13} \mathrm{C}(5)$ & ${ }^{13} \mathrm{C}(6)$ \\
\hline $\mathrm{A} / \mathrm{MHz}$ & $2709.4170(25)$ & $2740.5933(64)$ & $2749.4880(80)$ & $2706.2390(62)$ & $2736.6705(89)$ & $2747.6705(52)$ \\
$\mathrm{B} / \mathrm{MHz}$ & $1230.8059(12)$ & $1227.8970(15)$ & $1226.6421(13)$ & $1222.3991(15)$ & $1212.3571(19)$ & $1217.9319(13)$ \\
$\mathrm{C} / \mathrm{MHz}$ & $1070.4833(11)$ & $1073.9901(14)$ & $1073.3059(13)$ & $1066.7514(12)$ & $1064.6020(17)$ & $1067.0527(12)$ \\
& & & & & & \\
$\Delta_{\mathrm{J}} / \mathrm{kHz}$ & {$[0.359]$} & {$[0.359]$} & {$[0.359]$} & {$[0.359]$} & {$[0.359]$} & {$[0.359]$} \\
$\Delta_{\mathrm{JK}} /$ & {$[0.254]$} & {$[0.254]$} & {$[0.254]$} & {$[0.254]$} & {$[0.254]$} & {$[0.254]$} \\
$\mathrm{kHz}$ & & & & & & \\
$\Delta_{\mathrm{K}} / \mathrm{kHz}$ & {$[1.02]$} & {$[1.02]$} & {$[1.02]$} & {$[1.02]$} & {$[1.02]$} & {$[1.02]$} \\
$\delta_{\mathrm{J}} / \mathrm{kHz}$ & {$[-0.0132]$} & {$[-0.0132]$} & {$[-0.0132]$} & {$[-0.0132]$} & {$[-0.0132]$} & {$[-0.0132]$} \\
$\delta_{\mathrm{K}} / \mathrm{kHz}$ & {$[-1.88]$} & {$[-1.88]$} & {$[-1.88]$} & {$[-1.88]$} & {$[-1.88]$} & {$[-1.88]$} \\
& & & & & & 11 \\
$\mathrm{~N}_{\text {lines }}$ & 12 & 10 & 9 & 12 & 8.50 & 11 \\
$\sigma_{\text {fit }} / \mathrm{kHz}$ & 5.45 & 6.27 & 5.59 & 6.89 & 5.64 \\
\hline
\end{tabular}

Quartic centrifugal distortion constants in brackets were constrained to the values of the ${ }^{12} \mathrm{C} /{ }^{16} \mathrm{O} /{ }^{1} \mathrm{H}$ isotopologue.

Table S15. Experimental rotational parameters of the solketal single ${ }^{18} \mathrm{O}$ isotopologues.

\begin{tabular}{llll}
\hline & \multicolumn{1}{c}{${ }^{18} \mathrm{O}(1)$} & \multicolumn{1}{c}{${ }^{18} \mathrm{O}(2)$} & \multicolumn{1}{c}{${ }^{18} \mathrm{O}(3)$} \\
\hline $\mathrm{A} / \mathrm{MHz}$ & $2709.16(19)$ & $2728.49(19)$ & $2728.51(66)$ \\
$\mathrm{B} / \mathrm{MHz}$ & $1220.8886(15)$ & $1227.9991(15)$ & $1187.5827(42)$ \\
$\mathrm{C} / \mathrm{MHz}$ & $1069.3034(15)$ & $1075.8185(15)$ & $1040.4852(32)$ \\
& & & \\
$\Delta_{\mathrm{J}} / \mathrm{kHz}$ & {$[0.359]$} & {$[0.359]$} & {$[0.359]$} \\
$\Delta_{\mathrm{JK}} / \mathrm{kHz}$ & {$[0.254]$} & {$[0.254]$} & {$[0.254]$} \\
$\Delta_{\mathrm{K}} / \mathrm{kHz}$ & {$[1.02]$} & {$[1.02]$} & {$[1.02]$} \\
$\delta_{\mathrm{J}} / \mathrm{kHz}$ & {$[-0.0132]$} & {$[-0.0132]$} & {$[-0.0132]$} \\
$\delta_{\mathrm{K}} / \mathrm{kHz}$ & {$[-1.88]$} & {$[-1.88]$} & {$[-1.88]$} \\
& & & \\
$\mathrm{N}_{\text {lines }}$ & 7 & 6 & 6 \\
$\sigma_{\text {fit }} / \mathrm{kHz}$ & 5.56 & 5.11 & 9.40 \\
\hline
\end{tabular}

Quartic centrifugal distortion constants in brackets were constrained to the values of the ${ }^{12} \mathrm{C} /{ }^{16} \mathrm{O} /{ }^{1} \mathrm{H}$ isotopologue. 


\section{Kraitchman Analysis}

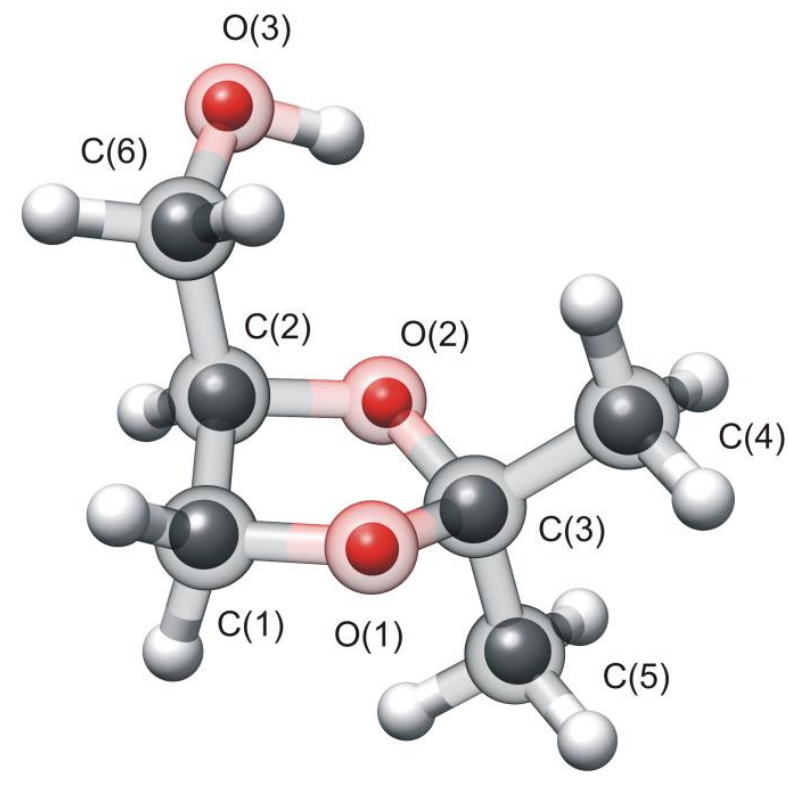

Figure S1. Atom labeling of solketal. Inner spheres represent atom positions obtained from the Kraitchman substitution coordinates. Transparent spheres and hydrogen atoms represent atom positions of the M06-2X/6-311++G(d,p) optimized structure.

Table S16. Substitution coordinates $\left(r_{s}\right)$ of the carbon and oxygen atoms from the general Kraitchman equations using the rotational constants of the single ${ }^{13} \mathrm{C}$ and ${ }^{18} \mathrm{O}$ isotopologues from Tables S14 and S15.

\begin{tabular}{lcccccc}
\hline & $\mathrm{a}$ & $\delta \mathrm{a}$ & $\mathrm{b}$ & $\delta \mathrm{b}$ & $\mathrm{c}$ & $\delta \mathrm{c}$ \\
\hline $\mathrm{C}(1)$ & $0.17096 \cdot \mathrm{i}^{*}$ & $0.00884 ?$ & 1.66203 & 0.00091 & 0.19443 & 0.00778 \\
$\mathrm{C}(2)$ & 0.86498 & 0.00176 & 0.66049 & 0.00230 & 0.48525 & 0.00313 \\
$\mathrm{C}(3)$ & 1.18802 & 0.00128 & 0.27691 & 0.00549 & $0.07512 \cdot i^{*}$ & $0.02023 ?$ \\
$\mathrm{C}(4)$ & 1.44261 & 0.00105 & 1.50937 & 0.00101 & 0.87680 & 0.00173 \\
$\mathrm{C}(5)$ & 2.30924 & 0.00066 & 0.06256 & 0.02448 & 0.97399 & 0.00157 \\
$\mathrm{C}(6)$ & 2.05250 & 0.00074 & 0.20973 & 0.00723 & 0.38949 & 0.00389 \\
$\mathrm{O}(1)$ & 0.97297 & 0.00229 & 0.82137 & 0.00270 & 0.87029 & 0.00261 \\
$\mathrm{O}(2)$ & $0.19934 \cdot i^{*}$ & $0.01111 ?$ & 0.47888 & 0.00459 & 0.72287 & 0.00308 \\
$\mathrm{O}(3)$ & 2.73834 & 0.00214 & 0.87927 & 0.00709 & 0.16522 & 0.03815 \\
\hline
\end{tabular}

* Set to zero for structure comparison shown in Figure S1. 


\section{References}

(1) C.H. Townes, A.L. Schawlow, Microwave Spectroscopy (Dover books on Physics), $2^{\text {nd }}$ ed. 2012.

(2) D. Patterson, J.M. Doyle, Sensitive Chiral Analysis via Microwave Three-Wave Mixing. Phys. Rev. Lett. 111, 023008 (2013).

(3) E. Hirota, Triple Resonance for a Three-Level System of a Chiral Molecule. Proc. Jpn. Acad. Ser. B 88, 120$128,(2012)$.

(4) M. Sargent, M.O. Scully, and W.E. Lamb, Laser Physics, Westview Press, 1974, Appendix A

(5) Gaussian 09, M. J. Frisch, G. W. Trucks, H. B. Schlegel, G. E. Scuseria, M. A. Robb, J. R. Cheeseman, G. Scalmani, V. Barone, B. Mennucci, G. A. Petersson, H. Nakatsuji, M. Caricato, X. Li, H. P. Hratchian, A. F. Izmaylov, J. Bloino, G. Zheng, J. L. Sonnenberg, M. Hada, M. Ehara, K. Toyota, R. Fukuda, J. Hasegawa, M. Ishida, T. Nakajima, Y. Honda, O. Kitao, H. Nakai, T. Vreven, J. A. Montgomery, Jr., J. E. Peralta, F. Ogliaro, M. Bearpark, J. J. Heyd, E. Brothers, K. N. Kudin, V. N. Staroverov, R. Kobayashi, J. Normand, K. Raghavachari, A. Rendell, J. C. Burant, S. S. Iyengar, J. Tomasi, M. Cossi, N. Rega, J. M. Millam, M. Klene, J. E. Knox, J. B. Cross, V. Bakken, C. Adamo, J. Jaramillo, R. Gomperts, R. E. Stratmann, O. Yazyev, A. J. Austin, R. Cammi, C. Pomelli, J. W. Ochterski, R. L. Martin, K. Morokuma, V. G. Zakrzewski, G. A. Voth, P. Salvador, J. J. Dannenberg, S. Dapprich, A. D. Daniels, Ö. Farkas, J. B. Foresman, J. V. Ortiz, J. Cioslowski, and D. J. Fox, Gaussian, Inc., Wallingford CT, 2009.

(6) D. Plusquellic, JB95, available at http://www.nist.gov/pml/div686/sources_detectors/jb95.cfm

(7) Z. Kisiel, L. Pszczółkowski, I. R. Medvedev, M. Winnewisser, F. C. De Lucia, E. Herbst, Rotational spectrum of trans-trans diethyl ether in the ground and three excited vibrational states, J. Mol. Spectrosc. 233, 231 (2005). doi:10.1016/j.jms.2005.07.006

(8) H. M. Pickett, The fitting and prediction of vibration-rotation spectra with spin interactions, J. Mol. Spectrosc. 148, 371 (1991). doi:10.1016/0022-2852(91)90393-O

(9) H. M. Pickett, SPFIT/SPCAT package, available at http://spec.jpl.nasa.gov

(10) Z. Kisiel, PROSPE - Programs for ROtational SPEctroscopy, available at www.ifpan.edu.pl/ kisiel/prospe.htm

(11) E.F. Pettersen, T. D. Goddard, C. C. Huang, G. S. Couch, D. M. Greenblatt, E. C. Meng, T. E. Ferrin,UCSF Chimera - A visualization system for exploratory research and analysis, J. Comput. Chem., 131605 (2004). doi: $10.1002 /$ jcc. 20084 